\title{
PERDA AUDITIVA INDUZIDA PELO RUÍDO EM SERVIDORES DE UMA UNIVERSIDADE ESTADUAL PÚBLICA
}

Camila Helen de Oliveira1', Maria do Carmo Lourenço Haddad², Mariana Angela Rossaneis³, Dayane Aparecida Scaramal!

${ }^{1}$ Enfermeira. Mestranda em Enfermagem. Universidade Estadual de Londrina. Londrina-PR-Brasil.

${ }^{2}$ Enfermeira. Doutora em Enfermagem. Docente na Universidade Estadual de Londrina. Londrina-PR-Brasil.

${ }^{3}$ Enfermeira. Doutoranda em Enfermagem. Universidade Estadual de Londrina. Londrina-PR-Brasil.

RESUMO: O objetivo desta pesquisa foi analisar a Perda Auditiva Induzida pelo Ruído em servidores de uma universidade estadual pública do norte do Paraná. A pesquisa se caracteriza como um estudo transversal descritivo, realizado entre junho e julho de 2014. Os dados foram obtidos através de análise documental. Participaram da pesquisa 131 trabalhadores com idade média de 53 anos e cada um realizou em média três exames audiométricos. Observou-se que na última audiometria realizada 30\% do grupo amostral apresentou algum tipo de perda auditiva, o que representa um aumento 2,3 vezes ou $230 \%$ de portadores deste agravo, ao comparar com a audiometria de referência. Estes resultados indicam que não basta a realização de exames audiométricos pontuais, mas ações de prevenção e promoção à saúde, incluindo o trabalhador no planejamento e controle de sua saúde auditiva.

DESCRITORES: Audiometria; Perda auditiva; Ruído; Saúde do trabalhador.

\section{NOISE INDUCED HEARING LOSS AMONG STAFF IN A PUBLIC STATE UNIVERSITY}

\begin{abstract}
This study aimed to analyze Noise Induced Hearing Loss among the staff of a public state university in the north of Paraná. The research is characterized as a descriptive transversal study, undertaken between June and July 2014. The data were obtained through documental analysis. A total of 131 workers participated in the research, with a mean age of 53 years old, each one undertaking an average of three audiometric examinations. It was observed that in the last audiometric examination undertaken, 30\% of the sample group presented some form of hearing loss, representing an increase of 2.3 times, or $230 \%$, of persons with this health problem, in comparison with the reference audiometric examination. These results indicate that it is not enough to undertake occasional audiometric examinations, but that actions for promoting health and preventing harm to health are also necessary, including the worker in the planning and control of her auditory health. DESCRIPTORS: Audiometry; Hearing loss; Noise; Occupational health.
\end{abstract}

\section{PÉRDIDA AUDITIVA INDUCIDA POR EL RUIDO EN FUNCIONARIOS DE UNA UNIVERSIDAD ESTADUAL PÚBLICA}

RESUMEN: El objetivo de esta investigación fue analizar la Pérdida Auditiva Inducida por el Ruido en funcionarios de una universidad estadual pública de norte de Paraná. La investigación se caracteriza como un estudio transversal descriptivo, realizado entre junio y julio de 2014. Los datos fueron obtenidos a través de análisis documental. Participaron de la investigación 131 trabajadores con edad media de 53 años y cada uno realizó en media tres exámenes audiométricos. Se observó que en la última audiometría realizada, 30\% del grupo de la muestra presentó algun tipo de pérdida auditiva, lo que representa un aumento 2,3 veces o $230 \%$ de portadores de este agravio, haciéndose una comparación con la audiometría de referencia. Estes resultados apuntan que no basta solo la realización de exámenes audiométricos pontuales, pero acciones de prevención y promoción de salud, incluyendo el trabajador en el planeamiento y control de su salud auditiva. DESCRIPTORES: Audiometría; Pérdida auditiva; Ruido; Salud del trabajador. 


\section{INTRODUÇÃO}

A Perda Auditiva Induzida pelo Ruído (PAIR) está entre os principais problemas de saúde do trabalhador brasileiro e ocupa o segundo lugar entre as doenças mais frequentes do aparelho auditivo $^{(1)}$.

O ruído é considerado o risco físico mais comum entre os trabalhadores da indústria, podendo levar à PAIR, um distúrbio irreversível, mas completamente previnível ${ }^{(2)}$. A exposição ao ruído em função da frequência e suas múltiplas consequências para o organismo humano constitui um dos principais problemas de saúde ocupacional e ambiental na atualidade ${ }^{(3)}$.

De acordo com o Anexo I da Norma Regulamentadora NR - 7, Portaria SIT (Secretaria de Inspeção do Trabalho) 223/2011, de 06 de maio de 2011, entende-se por PAIR as alterações dos limiares auditivos, do tipo neurossensorial, decorrente da exposição ocupacional sistemática a patamares de pressão sonora elevada. Tem como características principais a irreversibilidade e a progressão gradual com o tempo de exposição ao risCo $^{(4)}$.

A PAIR tem como sinônimos: perda auditiva por exposição ao ruído no trabalho, perda auditiva ocupacional, surdez profissional, disacusia ocupacional, perda auditiva induzida por níveis elevados de pressão sonora, perda auditiva induzida por ruído ocupacional, perda auditiva neurossensorial por exposição continuada a níveis elevados de pressão sonora de origem ocupacional(5).

Este tipo de perda auditiva tem como principais características: ser sempre do tipo neurossensorial, por afetar as células de Corti; ser irreversível e afetar quase sempre ambos ouvidos; manifestarse diante de intolerâncias a sons intensos e zumbidos, comprometendo a comunicação devido à dificuldade de compreensão da fala. A progressão do agravo cessa quando há interrupção na exposição ao ruído. Alguns fatores são capazes de influenciar diretamente a PAIR relacionada ao trabalho, como: características físicas do ruído (tipo, espectro e nível de pressão sonora), susceptibilidade individual e tempo de exposição( ${ }^{(5)}$.

Consideram-se doenças ocupacionais as patologias adquiridas decorrentes da atividade laboral, a Portaria n. 777, de 28 de abril de 2004 do Ministério da Saúde normatiza a notificação compulsória da PAIR ${ }^{(6)}$.

No Brasil, as políticas públicas voltadas para a saúde do trabalhador começaram a ser implementadas a partir de 2003. Entre as estratégias para efetivação da Atenção Integral a Saúde do Trabalhador destaca-se a Rede Nacional de Atenção Integral à Saúde do Trabalhador, cujos objetivos são unir a rede de serviços do Sistema Único de Saúde (SUS) direcionados ao atendimento prestado à vigilância e realizar a notificação de agravos à saúde ligados ao trabalho em redes de serviço sentinela(5). Segundo dados do Sistema de Informação de Agravos de Notificação (SINAN) entre 2007 a 2012 foram notificados 1872 casos de PAIR, com maior índice nos estados de São Paulo com 646 notificações, 296 em Minas Gerais e 255 no Rio de Janeiro ${ }^{(7)}$.

Os dados relativos à PAIR, ou a respeito da população exposta ao ruído, ainda são raros e os que estão disponíveis expressam, parcialmente, a ocorrência do risco relacionado à perda auditiva. Estas informações são altamente relevantes para a estimativa do número de trabalhadores vulneráveis e na identificação dos ramos de atividade mais acometidos, constituindo subsídios para as ações de vigilância e prevenção da $\operatorname{PAIR}^{(8)}$.

A PAIR compromete a qualidade de vida do trabalhador devido a alterações funcionais e psicossociais ${ }^{(6)}$. Portanto é imprescindível que se desenvolvam mais ações de controle e prevenção de seus riscos e agravos. A World Health Organization - $\mathrm{WHO}^{(9)}$ e o Ministério da Saúde ${ }^{(5)}$ concordam que a PAIR e aos acidentes de trabalho são decorrentes também de prática individual que pode ser extinta, se houver ações de fiscalização, orientação e intervenção no ambiente de trabalho.

Com este cenário, o objetivo deste estudo foi analisar a perda auditiva induzida pelo ruído em servidores de uma universidade estadual pública.

\section{MÉTODO}

Trata-se de um estudo descritivo, transversal, de abordagem quantitativa. A pesquisa foi desenvolvida no Serviço Especializado em Engenharia de Segurança e Medicina do Trabalho (SESMT), do campus de uma universidade estadual pública do Paraná, no ano de 2014. 
Este serviço é composto por dois técnicos, um enfermeiro, um engenheiro e um médico do trabalho e tem como principal responsabilidade promover e proteger a integridade de 5.353 trabalhadores no seu local de trabalho de acordo com a Norma Regulamentador NR4 ${ }^{(10)}$.

A população deste estudo foi constituída por prontuários de trabalhadores, servidores contratados pelo Regime Jurídico Único dos Servidores Públicos do Estado do Paraná, que possuíam risco de PAIR devido às características da sua função, ou seja, trabalhavam em um ambiente onde o ruído ultrapassava os $85 \mathrm{~dB}$ e, por esse motivo tinham o exame audiométrico incluído em seu exame ocupacional periódico. Foram incluídos neste estudo: trabalhadores que exerciam atividades em ambientes cujos níveis de pressão sonora ultrapassem os limites de tolerância estabelecidos pelo Ministério de Trabalho, independente do uso de protetor auditivo $^{(4)}$; possuir exame audiométrico de referência e o sequencial. O exame audiométrico de referência é a primeira avaliação audiométrica realizada após a admissão do trabalhador no ambiente onde o ruído constitui-se um risco ocupacional. O exame audiométrico sequencial é aquele que será comparado com o de referência ${ }^{(4)}$. O critério de exclusão esteve relacionado à existência de distúrbios de audição conhecidos anteriormente ao ingresso do trabalhador na instituição. Esse dado foi obtido através do exame médico admissional ou em consultas médicas subsequentes documentadas no prontuário ocupacional disponível no serviço.

De acordo com os critérios, 231 prontuários se enquadravam para análise, porém foram selecionados 131, uma vez que 30 prontuários não possuíam nenhuma audiometria; 53 submetidos a apenas ao exame audiométrico de referência; cinco por terem diagnóstico de trauma acústico; seis por apresentarem patologias que comprometem o aparelho auditivo e seis com audiometrias com laudos inconclusivos ou interrogados, nos quais não foi possível identificar o resultado do exame.

Para a coleta de dados foi elaborada uma planilha no programa Microsoft Excel 2010 contendo as seguintes variáveis: número de prontuário, sexo, idade, tempo de atuação na instituição, função, resultado dos exames audiométricos de referência e sequencial, conduta no exame periódico ocupacional, resultados e condutas em audiometrias anteriores, se houvesse.

Todos os exames audiométricos foram realizados pelo serviço de fonoaudiologia da própria instituição, atendendo as especificações estabelecidas pela Portaria n. 19 da NR-7, como: uso de cabine acústica, equipamento devidamente calibrado, orientação de repouso acústico de 14 horas e realização do exame por profissional qualificado neste caso, o fonoaudiólogo ${ }^{(5)}$.

A análise dos dados foi realizada a partir da utilização do pacote estatístico Statistical Package for Social Sciences (SPSS) 20.0. Este estudo foi submetido e aprovado pelo Comitê de Ética em Pesquisa com Seres Humanos com CAAE: 21405214.7.0000.5231.

\section{RESULTADOS}

Neste estudo, dentre os 131 trabalhadores, $63 \%$ eram do sexo masculino; e, de acordo com a distribuição por faixa etária, houve maior concentração entre 52 e 61 anos (49\%), bem como entre 42 e 51 anos (37\%), a média das idades foi de 52 anos e a mediana 53 anos.

No que refere à lotação dos trabalhadores, $52,6 \%$ eram da Casa de Cultura, com predominância da categoria de músicos, que representaram $36 \%$ do total da amostra. O segundo local com maior lotação de servidores expostos ao PAIR foi o restaurante universitário $(18,3 \%)$ e a categoria de trabalhadores em maior quantidade nesse setor era dos cozinheiros (7\%) (Tabela 1). O tempo de serviço dos profissionais na instituição variou entre cinco e 42 anos, com média igual a 22 anos e mediana de 21 anos.

Os resultados do exame audiométrico de referência identificaram que 13 indivíduos possuíam o diagnóstico de PAIR bilateral $(10 \%)$, quatro tinham PAIR unilateral (3\%) e 36 $(27 \%)$ apresentaram rebaixamento dos limiares auditivos. Os outros 78 trabalhadores (60\%) possuíam resultado do exame dentro dos padrões de normalidade (Tabela 2). A Casa de Cultura foi o setor que apresentou servidores com as audiometrias iniciais mais comprometidas (8,7\%).

Os resultados do exameaudiométricosequencial mostraram que 31 indivíduos (24\%) apresentaram PAIR bilateral, oito (6\%) PAIR unilateral e 35 (26\%) possuíam rebaixamento auditivo (Tabela 3). 
Em média, cada trabalhador realizou três audiometrias ao longo do tempo de trabalho na instituição, sendo que nesse estudo foram analisadas a audiometria de referencia e a última audiometria sequencial. $\mathrm{O}$ intervalo médio entre elas foi de 10 anos.

$\mathrm{Na}$ comparação dos resultados das audiometrias identificou-se que na audiometria de referência 17 trabalhadores (13\%) possuíam o diagnóstico de PAIR (unilateral e bilateral) e na audiometria sequencial houve um aumento dos profissionais acometidos pela patologia, sendo que este quantitativo passou a ser de 39 servidores (30\%). Sendo assim, verificou-se um aumento de 2,3 vezes (230\%) no número de trabalhadores acometidos por PAIR na instituição em estudo, no período entre a audiometria de referência e a última audiometria sequencial.

Também foi possível avaliar no registro dos exames periódicos ocupacionais as principais orientações e condutas realizadas a estes servidores, no qual se constatou que em 59\% dos prontuários não havia anotações sobre orientações ou esclarecimentos relacionados à prevenção ou ao tratamento da PAIR.

Em relação às orientações, apenas em $26 \%$ foram identificados registros de informações relativas ao uso de Equipamentos de Proteção Individual (EPI), neste caso o uso de protetor auricular; $10 \%$ foram encaminhados ao otorrinolaringologista e $7 \%$ foram orientados a utilizar solução otológica para remoção de rolha de cerume, seguido de $5 \%$ que foram orientados a se abster de locais com ruídos intensos, utilizar prótese auricular, praticar lavagem auricular sempre que necessário e solicitar revisão do Programa de Prevenção de Riscos Ambientais (PPRA) do serviço em que o trabalhador estava lotado.
Tabela 1 - Distribuição de trabalhadores em risco de desenvolver a PAIR relacionada ao trabalho por setores e funçãoem uma universidade estadual pública. Londrina-PR, 2014.

\begin{tabular}{|c|c|c|}
\hline $\begin{array}{l}\text { Setores / } \\
\text { Função dos trabalhadores }\end{array}$ & N (131) & $\%$ \\
\hline Casa de cultura & 69 & 52,6 \\
\hline Arquivista de orquestra & 01 & 0,8 \\
\hline $\begin{array}{l}\text { Auxiliar operacional/ } \\
\text { ajudante geral }\end{array}$ & 07 & 5,3 \\
\hline Inspetor de orquestra & 01 & 0,8 \\
\hline Músicos & 47 & 36 \\
\hline $\begin{array}{l}\text { Operador de som e } \\
\text { imagem }\end{array}$ & 02 & 1,5 \\
\hline Técnico administrativo & 07 & 5,3 \\
\hline $\begin{array}{l}\text { Técnico em montagem de } \\
\text { eventos }\end{array}$ & 04 & 3 \\
\hline Restaurante universitário & 23 & 18,3 \\
\hline $\begin{array}{l}\text { Auxiliar operacional/ } \\
\text { ajudante geral }\end{array}$ & 06 & 5,3 \\
\hline Auxiliar de cozinha & 05 & 4 \\
\hline Cozinheiro & 09 & 7 \\
\hline Nutricionista & 01 & 0,8 \\
\hline Zelador & 01 & 0,8 \\
\hline $\begin{array}{l}\text { Prefeitura do campus } \\
\text { universitário }\end{array}$ & 19 & 14,5 \\
\hline $\begin{array}{l}\text { Auxiliar operacional/ } \\
\text { ajudante geral }\end{array}$ & 01 & 0,8 \\
\hline Jardineiro & 08 & 6 \\
\hline Marceneiro & 02 & 1,5 \\
\hline $\begin{array}{l}\text { Motorista de máquina } \\
\text { pesada }\end{array}$ & 01 & 0,8 \\
\hline Oficial de manutenção & 02 & 1,5 \\
\hline Pedreiro & 01 & 0,8 \\
\hline Serralheiro & 01 & 0,8 \\
\hline Telefonista & 03 & 2,3 \\
\hline Rádio universitário & 13 & 10 \\
\hline Chefe de setor & 01 & 0,8 \\
\hline $\begin{array}{l}\text { Comunicador social/ } \\
\text { jornalista }\end{array}$ & 04 & 3 \\
\hline Técnico administrativo & 01 & 0,8 \\
\hline $\begin{array}{l}\text { Técnico de estúdio e } \\
\text { multimídia }\end{array}$ & 07 & 5,3 \\
\hline Serviços gráficos & 07 & 4,6 \\
\hline Auxiliar administrativo & 01 & 0,8 \\
\hline Chefe de setor & 01 & 0,8 \\
\hline Operador gráfico & 04 & 3 \\
\hline
\end{tabular}


Tabela 2 - Resultados das audiometrias de referência de servidores em risco de desenvolverem PAIR, de acordo com os setores ocupacionais de uma universidade estadual pública. Londrina-PR, 2014.

\begin{tabular}{|c|c|c|c|c|c|c|c|c|c|c|}
\hline \multirow{3}{*}{ Setores } & \multicolumn{10}{|c|}{ Resultados das audiometrias } \\
\hline & \multicolumn{2}{|c|}{ PAIR bilateral } & \multicolumn{2}{|c|}{ PAIR unilateral } & \multicolumn{2}{|c|}{$\begin{array}{c}\text { Rebaixamento } \\
\text { auditivo }\end{array}$} & \multicolumn{2}{|c|}{ Normal } & \multicolumn{2}{|c|}{ Total } \\
\hline & $\mathrm{N}$ & $\%$ & $\mathrm{~N}$ & $\%$ & $\mathrm{~N}$ & $\%$ & $\mathrm{~N}$ & $\%$ & $\mathrm{~N}$ & $\%$ \\
\hline Casa de Cultura & 05 & 7,2 & 01 & 1,5 & 17 & 24,6 & 46 & 66,7 & 69 & 52,6 \\
\hline Prefeitura do campus & 03 & 15,7 & 00 & 0 & 07 & 37 & 09 & 47,3 & 19 & 18,3 \\
\hline Rádio universidade & 02 & 15,4 & 01 & 7,8 & 03 & 23 & 07 & 53,8 & 13 & 10 \\
\hline Restaurante universitário & 02 & 8,7 & 02 & 8,7 & 06 & 26,1 & 13 & 56,5 & 23 & 18,3 \\
\hline Serviços gráficos & 01 & 14,4 & 00 & 0 & 03 & 42,8 & 03 & 42,8 & 07 & 4,6 \\
\hline Total & 13 & 10 & 04 & 3 & 36 & 27 & 78 & 60 & 131 & 100 \\
\hline
\end{tabular}

Tabela 3 - Resultados da última audiometria sequencial de servidores em risco de desenvolver PAIR, de acordo com os setores de uma universidade estadual pública. Londrina-PR, 2014.

\begin{tabular}{|c|c|c|c|c|c|c|c|c|c|c|}
\hline \multirow{3}{*}{ Setores } & \multicolumn{10}{|c|}{ Resultados da última audiometria sequencial } \\
\hline & \multicolumn{2}{|c|}{ PAIR bilateral } & \multicolumn{2}{|c|}{ PAIR unilateral } & \multicolumn{2}{|c|}{$\begin{array}{l}\text { Rebaixamento } \\
\text { auditivo }\end{array}$} & \multicolumn{2}{|c|}{ Normal } & \multicolumn{2}{|c|}{ Total } \\
\hline & $\mathrm{N}$ & $\%$ & $\mathrm{~N}$ & $\%$ & $\mathrm{~N}$ & $\%$ & $\mathrm{~N}$ & $\%$ & $\mathrm{~N}$ & $\%$ \\
\hline Casa de Cultura & 15 & 21,7 & 03 & 4,3 & 20 & 29 & 31 & 45 & 69 & 52,6 \\
\hline Prefeitura do campus & 06 & 31,6 & 03 & 15,8 & 02 & 10,5 & 08 & 42,1 & 19 & 18,3 \\
\hline Rádio universidade & 02 & 15,4 & 01 & 7,7 & 04 & 30,8 & 06 & 46,1 & 13 & 10 \\
\hline Restaurante universitário & 07 & 30,4 & 01 & 4,4 & 08 & 34,8 & 07 & 30,4 & 23 & 18,3 \\
\hline Serviços gráficos & 01 & 14,3 & 0 & 0 & 01 & 14,3 & 05 & 71,4 & 07 & 4,6 \\
\hline Total & 31 & 24 & 08 & 06 & 35 & 26 & 57 & 44 & 131 & 100 \\
\hline
\end{tabular}

\section{DISCUSSÃO}

O ruído ocupacional é o principal causador de PAIR, zumbido e mudança no limiar auditivo, resultando em problemas na comunicação e dificuldade nas interações sociais. Este fenômeno também provoca efeitos secundários como o estresse e a dificuldade em estabelecer comunicação eficaz no trabalho, provocando acidentes $^{(11)}$.

Neste estudo foi possível observar que houve um aumento dos casos de PAIR em trabalhadores com mediana de 52 anos de idade e que atuam em média há 22 anos na instituição, podendo sugerir que há uma relação entre estas variáveis. Corroborando com este achado, um estudo destaca o aumento da prevalência de perda auditiva com o avançar da idade, chegando a ser 11,45 vezes mais elevada na faixa etária acima de 50 anos, quando comparados com aqueles com idade inferior a 30 anos. Este mesmo estudo observou ainda a relação de PAIR com o tempo de atividade na empresa, constatando aumento da prevalência dos casos sugestivos de perda auditiva, a partir de seis anos de atividade laboral|(12).

Esta inferência pôde ser realizada por se tratar de servidores estaduais, admitidos por meio de concurso público e que possuem como característica estabilidade do vínculo empregatício.

A prevalência de PAIR observada neste estudo foi de $30 \%$, correspondente à perda da audição unilateral e bilateral encontrada no último exame audiométrico realizado pelo servidor e está ligeiramente mais elevada que o estimado pelo Ministério da Saúde ${ }^{(5)} q u e$ é de 25\% na população exposta ao ruído ocupacional. Em um estudo realizado com trabalhadores em diversas funções industriais $46 \%$ apresentavam algum tipo de PAIR unilateral ou bilateral ${ }^{(13)}$.

Já em outro estudo com população semelhante, a prevalência encontrada foi de $35,7 \%$ entre aqueles com atividades no ramo da construção civil, marcenaria e jardinagem ${ }^{(14)}$. Ressalta-se que há maior risco de PAIR entre os trabalhadores 
da construção civil, pois ao contrário do que acontece no setor industrial, o ruído não tem diminuído com o passar dos anos. Faltam investimentos em modernização das máquinas e no cuidado com a saúde ocupacional ${ }^{(15)}$.

Identificou-se ainda uma alta prevalência do PAIR em músicos. Em um estudo comparativo entre músicos militares e um grupo controle, a prevalência de PAIR encontrada nestes profissionais foi de $32 \%$, significando que esta categoria apresentou um risco de 14,54 vezes maior de desenvolver algum tipo de alteração auditiva do que indivíduos sem histórico de exposição à música ${ }^{(16)}$. Em pesquisa semelhante foi encontrado $17 \%$ de prevalência de PAIR, também demonstrando uma correlação positiva para tempo de atuação profissional e uma piora nos limiares audiométricos ${ }^{(17)}$.

No caso dos músicos profissionais nesta pesquisa, a prevalência de PAIR ficou próxima à encontrada nos estudos descritos acima. As elevadas taxas de perda auditiva nesta categoria profissional parecem determinadas por fatores extrínsecos e intrínsecos. São considerados fatores externos: o tempo de exposição ao ruído em anos, a posição ocupada pelo músico no palco: fosso de orquestra ou perto de altofalantes, bem como o tipo instrumento que toca. Já os fatores intrínsecos se relacionam com as diferenças psicofisiológicas e individuais ${ }^{(18)}$.

Em relação aos trabalhadores do restaurante universitário observou- se nesta pesquisa elevada porcentagem de trabalhadores acometidos pela PAIR. Um estudo realizado com trabalhadores de uma cozinha hospitalar revela que o risco de lesão auditiva parece estar associado ao ruído produzido na área de preparação de alimentos (principalmente de liquidificadores e utensílios), e do contato metalmetal entre panelas de aço inoxidável e prateleiras de metal. Os cozinheiros são expostos a muitas fontes de ruído de impacto intermitentes, tais como, o contato metal-metal entre utensílios e do uso de misturadores de tamanho industrial ${ }^{(19)}$.

Contudo, nesta análise documental, não foi possível identificar ações institucionais relacionadas à prevenção e ao controle da PAIR, mas os dados apresentados revelaram o crescimento do número de trabalhadores afetados no intervalo entre a audiometria de referência e a última realizada pelo servidor. Evidenciouse, portanto, que não basta realizar o exame audiométrico de acordo com o estabelecido é necessário e urgente estabelecer planos eficazes capazes de transformar o trabalhador em participante ativo e corresponsável pela sua saúde no ambiente de trabalho.

O relatório sobre o estado de saúde ocupacional da União Europeia traz a importância na implantação de medidas institucionais para a redução do ruído, como a modernização do maquinário e automação do processo de trabalho, estas ações estão mais voltadas ao setor industrial, já no setor da construção civil a introdução de novas ferramentas e equipamentos de trabalho foi identificada como tendo contribuído para o aumento dos níveis de ruído, sendo que a falta de informação, supervisão e controle dos riscos no ambiente de trabalho deixa o trabalhador mais vulnerável a PAIR ${ }^{(11)}$.

\section{CONCLUSÃO}

A prevalência de PAIR entre os trabalhadores foi de $30 \%$, somando a perda auditiva bilateral e unilateral, encontrada no último exame audiométrico da amostra estudada. Foi possível observar um aumento de 2,3 vezes ou $230 \%$ desta prevalência quando comparado ao resultado da audiometria de referência.

Observou-se que,em alguns setores ocupacionais, havia profissões mais acometidas por esta doença, como os músicos e os trabalhadores da cozinha. O tempo de exposição ao ruído e a idade podem contribuir no comprometimento auditivo. Dessa forma, com a alta prevalência de PAIR, nos diversos setores analisados evidenciada neste estudo, é possível inferir que há necessidade de um programa de prevenção e controle efetivo da PAIR na instituição.

Por fim, com o cumprimento das normas preconizadas pelo Ministério da Saúde, como a notificação obrigatória deste agravo, será possível conhecer a prevalência e obter subsídios para a construção de indicadores que norteiem o planejamento de ações eficazes de controle e prevenção da PAIR.

\section{REFERÊNCIAS}

1. Lopes AC, Otowiz VG, Lopes PMB, Lauris JRP, Santos CC. Prevalência de perda auditiva induzida por ruído em motoristas. Int. Arch. Otorhinolaryngol. [Internet] 
2012;16(4). [acesso em 20 mar 2014] 1. Disponível: http://dx.doi.org/10.7162/S1809-97772012000400013

2. Mostaghaci M, Mirmohammadi SJ, Mehrparvar AH, Bahaloo M, Mollasadeghi A, Davari MH. Effect of workplace noise on hearing ability in tile and ceramic industry workers in Iran: A 2-Year follow-up study. Hindawi Publishing Corporation The Scientific World Journal. [Internet] 2013;01 [acesso em 01 dez 2014]. Disponível: http://dx.doi.org/10.1155/2013/923731

3. BRASIL. Ministério da Saúde. Organização PanAmericana da Saúde no Brasil. Doenças relacionadas ao trabalhado: manual de procedimentos para os serviços de saúde. Brasília: Ministério da Saúde, 2001. p.580.

4. BRASIL. Secretaria de Segurança do Trabalho. Portaria SIT $n^{\circ} 06 / 2011$. Dispõe sobre o uso de equipamento de proteção individual. . [Internet]. 2011. [acesso em 01 dez 2014] Disponível: http://portal.mte.gov.br/ data/files/FF8080812DC56F8F012DCDAD35721F50/ NR-06\%20\%28atualizada\%29\%202010.pdf

5. BRASIL. Ministério da Saúde, Secretaria de Atenção à Saúde, Departamento de Ações Programáticas Estratégicas. Perda auditiva induzida por ruído (Pair). Ministério da Saúde, Brasília: Editora do Ministério da Saúde, 2006. p. 40

6. BRASIL. Ministério da Saúde. Portaria n 777, de 28 de abril de 2004. Dispõe sobre os procedimentos técnicos para a notificação compulsória de agravos à saúde do trabalhador em rede de serviços sentinela específica, no Sistema Único de Saúde (SUS). Diário Oficial da União, Brasília, DF, 28 abr. 2004. [Internet]. 2004. [acesso em 21 mar 2014]. Disponível: http://dtr2001.saude. gov.br/sas/PORTARIAS/Port2004/GM/GM-777.htm

7. Brasil. Ministério da Saúde. Centro colaborador da vigilância dos agravos à saúde relacionados ao trabalho. Perda Auditiva Induzida pelo Ruído. [Internet]. 2013. [acesso em 14 abr 2014]. Disponível: http://www.acm.org.br/acm/acamt/documentos/ vigilancia-pair-11-2013.pdf

8. Cavalcante F, Ferrite S, Costa Meira TC. Exposure to noise in the manufacturing industry in Brazil. Rev. CEFAC. [Internet]. 2013;15(5). [acesso em $03 \mathrm{dez}$ 2014]. Disponível: http://dx.doi.org/10.1590/S151618462013005000021

9. WHO - World Health Organization. Report on informal consultation on epidemiology of deafness and hearing impairment in developing countries and update of the WHO protocol. Geneva: WHO; 2004.

10. BRASIL. Secretaria de Segurança do Trabalho. Portaria GM n. ${ }^{\circ}$ 3.214, de 08 de junho de 1978, atualizada pela Portaria MTE n. ${ }^{0}$ 590, de 28 de abril de 2014). Dispõe sobre serviços especializados em engenharia de segurança e em medicina do Trabalho. [Internet]. 2011 [acesso em $01 \mathrm{dez}$
2014]. Disponível: http://portal.mte.gov.br/data/ files/FF80808145B269620145D2D2CC874DCC/ NR-04\%20(Atualizada\%202014).pdf

11. European Agency for Safety and Health at Work. Monitoring the state of occupational safety and health in the European Union - Pilot Study. [Internet] 2000. [acesso em 02 dez 2014]. Disponível: https://osha. europa.eu/en/publications/reports/401

12. Ribeiro Guerra MR, Lourenço PMC, BustamanteTeixeira MT, Alves MJM. Prevalência de perda auditiva induzida por ruído em empresa metalúrgica. Rev. Saude Publica. [Internet]. 2005;39(2) [acesso em 03 dez 2014]. Disponível: http://dx.doi.org/10.1590/ S0034-89102005000200015

13. Otoni A, Escher MB, Barbosa-Branco A, Eri HS, Alves MM. Ruído ocupacional como fator de risco para perda auditiva. Cogitare enferm. [Internet]. 2008;13(3) [acesso em 20 set 2014]. Disponível: http://ojs.c3sl.ufpr. br/ojs2/index.php/cogitare/article/view/12968/8763

14. Leão RN, Dias FAM. Perfil audiométrico de indivíduos expostos ao ruído atendidos no núcleo de saúde ocupacional de um hospital do município de Montes Claros, Minas Gerais. Rev. CEFAC [Internet]. 2010;12(2) [acesso em 11 abr 2014]. Disponível: http://dx.doi. org/10.1590/S1516-18462010000200010

15. Neitzel RL, Stover B, Seixas NS. Longitudinal assessment of noise exposure in a cohort of construction workers. Ann. Occup. Hyg. [Internet]. 2011;55(8) [acesso em 03 dez 2014]. Disponível: http://www.ncbi.nlm.nih. gov/pmc/articles/PMC3243919/pdf/mer050.pdf

16. Gonçalves CGO, Lacerda ABM, Zeigelboim BS, Marques JM, Luders D. Limiares auditivos em músicos militares: convencionais e altas frequências. CoDAS [Internet]. 2013;25(2) [acesso em 2014 abr 25]. Disponível: http://dx.doi.org/10.1590/S2317-17822013000200015

17. Amorim RB, Lopes AC, Santos KTP, Melo ADP, Lauris JRP. Auditory alterations for occupational exposition in musicians . Int. Arch. Otorhinolaryngol. 2008;12(3) [acesso em 05 mai 2014]. Disponível: http://www. arquivosdeorl.org.br/conteudo/acervo_eng.asp?ld=544

18. Schink T, Kreutz G, Busch V, Pigeot I, Ahrens W. Incidence and relative risk of hearing disorders in professional musicians. Occup Environ Med. [Internet]. 2014;71 [acesso em 04 dez 2014]. Disponível: http:// www.ncbi.nlm.nih.gov/pmc/articles/PMC4078669/ pdf/oemed-2014-102172.pdf

19. Achutan C. Assessment of noise exposure in a hospital kitchen. Noise \& Health [Internet]. 2009;11(44) [acesso em 04 dez 2014]. Disponível: http://www. noiseandhealth.org/article.asp?issn=1463-1741; year= 2009; volume $=11$; issue =44; spage $=145$; epage $=150 ;$ aul ast $=$ Achutan 